\title{
Archéopages
}

Archéopages

Archéologie et société

Hors-série 3 | 2012

Nouveaux champs de la recherche archéologique

\section{Traces de vie des derniers chasseurs-cueilleurs mésolithiques}

Frédéric Séara

\section{(2) OpenEdition}

1 Journals

Édition électronique

URL : https://journals.openedition.org/archeopages/699

DOI : 10.4000/archeopages.699

ISSN : 2269-9872

Éditeur

INRAP - Institut national de recherches archéologiques préventives

Édition imprimée

Date de publication : 1 janvier 2012

Pagination : 124-133

ISSN : 1622-8545

\section{Référence électronique}

Frédéric Séara, "Traces de vie des derniers chasseurs-cueilleurs mésolithiques », Archéopages [En ligne], Hors-série 3 | 2012, mis en ligne le 01 janvier 2012, consulté le 23 février 2023. URL : http:// journals.openedition.org/archeopages/699; DOI : https://doi.org/10.4000/archeopages.699 
à Clermont-Ferrand Puy-de-Dôme ", in DARTEvelle H. (DIR.), Auvergne et Midi, Actes des $5 e$ Rencontres Méridionales de

Préhistoire Récente, Clermont-Ferrand, novembre 2002, Cressenac, éd. Préhistoire du Sud-Ouest, p. 43-84.

VAllat P., 2008 : « Les Martres-D'Artière, Champ Chalatras », Bilan scientifique de la région d'Auvergne 2008, SRA, Paris, Ministère de la Culture, p. 120-121.

VAQUER J. 1990 : Le Néolithique en Languedoc Occidental, Paris, éd. CNRS, $397 \mathrm{p}$.

VAQUER J., 1998 : « Fortifications et pouvoir au Néolithique », L'Archéologue, $\mathrm{n}^{\circ}$ 35, p. 31-34, 9 fig.

Weller O., 2006 : « Prospection thématique. L'exploitation des sources salées en Auvergne entre Néolithique et Âge du Fer ", Bilan scientifique de la région d'Auvergne 2006, SRA, Paris, Ministère de la Culture, p. 158-159.

\section{Traces de vie des derniers chasseurs-cueilleurs mésolithiques}

Frédéric Séara

Inrap, UMR 7041 "Archéologies et Sciences de l'Antiquité»

C es dix dernières années ont connu une augmentation significative du nombre de gisements de plein air mésolithiques découverts, ce qui apparaît de toute évidence comme l'une des conséquences directes du développement de l'archéologie préventive. Toutefois, l'effectif de sites identifiés reste encore modeste si on le compare à celui atteint pour les périodes du Paléolithique et du Néolithique. Sur un plan géographique, une assez forte disparité existe, avec une moitié Nord de la France qui apparaît bien mieux placée pour la détection et la fouille de ce type d'occupation, principalement en raison de l'existence de contextes de conservation globalement plus favorables [Fig.1].

Dire que les opérations d'archéologie préventives ont permis de renouveler notre perception de la civilisation mésolithique pourrait paraître prétentieux, mais il faut admettre que de nombreux domaines d'étude (bien souvent à caractère palethnographique) y ont trouvé matière, ce qui constitue un préalable très prometteur face à l'objectif palethnologique. Bien entendu tout cela n'aurait pu se mettre en place aussi rapidement sans l'existence de travaux précurseurs tels que ceux du Dr J-G. Rozoy (Rozoy, 1978), de J. Hinout (Hinout, 1990), ou d'André Thévenin (Thévenin, 2008), qui se sont attachés à défendre l'étude d'une civilisation restée trop longtemps en marge des orientations données à la recherche archéologique.

1 Responsable d'opération, Frédéric Jallet (Inrap)

2 Responsable

d'opération, Émilie Millet (Inrap) ; fouille du secteur mésolithique, Sylvain Griselin et Bénédicte Souffi (Inrap). 3 Fouilles Laurent Lang, 2002. plus restreintes confirment également le caractère privilégié de ce milieu alluvial. Ces recherches ont abouti à une modification des schémas d'occupation construits à partir des fouilles conduites principalement dans des contextes sédimentaires sableux, majoritaires dans la région emblématique du Tardenois (Rozoy, 1978).

Lévolution du regard porté à la potentialité archéologique des différents contextes géomorphologiques constitue l'une des conséquences du développement de l'archéologie préventive et, en particulier, de la généralisation des moyens mécanisés qui l'accompagne. Cette condition technique reste indissociable du paramètre des compétences qui conditionne la qualité de réponse aux différents enjeux archéologiques, ainsi que leur niveau d'intégration dans les différentes étapes du volet opérationnel.

Au travers des données stratigraphiques, de nombreuses similitudes apparaissent. Ainsi la possibilité de définir des cadres stratigraphiques types s'explique par l'existence de dynamiques alluviales assez proches qui, tout en procurant des conditions favorables à l'installation des hommes du Mésolithique, ont assuré la conservation de leurs vestiges d'occupation. Les séquences stratigraphiques des grandes plaines alluviales témoignent de conditions de sédimentation qui sont globalement favorables à la conservation des occupations dès la fin du Tardiglaciaire, tout du moins pour la moitié nord de la France. Les analogies stratigraphiques sont, pour l'essentiel, le reflet de dynamiques alluviales très proches, à l'origine d'un taux d'aggradation d'intensité variable, mais constant (Séara et al., 2002). Aussi, même si le contexte alluvial paraît globalement privilégié, ce constat ne peut être généralisé à toutes les situations topographiques de fond de vallée.

Cependant, déterminer, très en amont, les conditions indispensables à l'existence d'un potentiel de conservation des occupations est un objectif réaliste qui, tout en orientant certains paramètres de la détection, n'en garantit pas le résultat, puisque le facteur premier est que l'homme se soit installé dans ces fonds de vallée. Les prospections pédestres, ont depuis longtemps démontré que bien d'autres contextes avaient été investis par les groupes mésolithiques, ce qui donne l'image d'un peuplement largement tourné hors du contexte alluvial (occupations de plateaux, marges du contexte alluvial, milieu karstique etc.). Des contextes particuliers de gisement ont permis de documenter, dans un cadre préventif, ces occupations situées à l'écart des fonds de vallée comme à Lhéry (Bostyn, Séara, 2011) et à Rosnay ${ }^{2}$ dans la Marne ou encore à Saint-Romain-sur-Cher dans le Loir-et-Cher (Kildéa, 2008a) et Langeais en Indre et Loire ${ }^{3}$ pour n'en citer que quelques-unes.

Ce constat, qui souligne le fort impact du biais taphonomique sur notre perception du peuplement mésolithique, pose la question de la part réelle du tropisme des cours d'eau face à la question des stratégies d'implantation.
Le contexte alluvial, un milieu privilégié. Le fait que la majorité des occupations mésolithiques de plein air détectées se trouve en fond de vallée ce milieu. Cela est, certes, évoqué par des sites " majeurs », tels que ceux de Ruffey-sur-Seille, Marpain dans le Jura (Séara, Roncin, 2010), d Warluis dans l'Oise (Ducrocq et al., 2008), de Rueil-Malmaison dans Les Hauts-de-Seine (Lang, Siccard, 2008) ou encore de Paris (Souffi, Marti, 2011) et de Lyon ${ }^{1}$, mais de nombreuses occupations

\section{des stratégies dimplantation.}




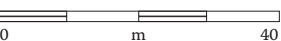

-.-. limite de fouille

= paléochenal

Wris Mésolithique récent

Mésolithique moyen

Mésolithique ancien/moyen

Mésolithique ancien

[Fig.2] Ruffey-sur-Seille (Jura). Plan de l'ensemble des vestiges distingués par phase chronologique. On constate la quasi-absence de superposition des phases d'occupation, ce qui constitue un schéma très particulier lié aux

modifications du milieu. La présence de vestiges sur cette surface de 25.000 $\mathrm{m}^{2}$ permet d'extrapoler cette configuration sur une très grande portion de la plaine alluviale de la rivière la Seille.

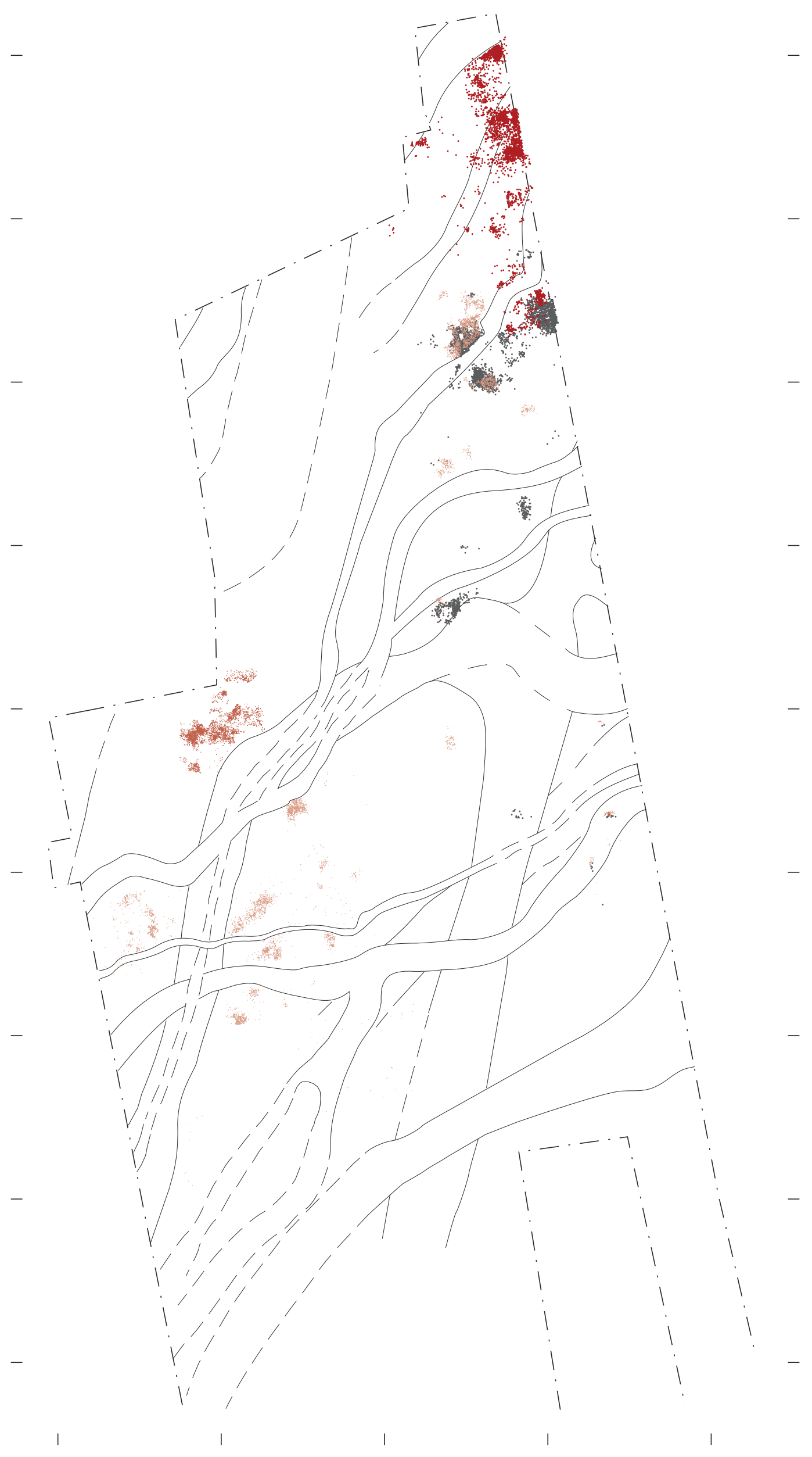


Détection, analyse et caractérisation : une réflexion de tous les instants. Le volet méthodologique a largement contribué à ce renouveau des connaissances. La détection des sites reste l'étape déterminante car, au-delà du seul fait d'arriver à détecter des occupations souvent très ténues, elle conditionne la nature des étapes ultérieures. La nature des gisements et des vestiges implique bien souvent des compétences particulières. Celles-ci sont nécessaires en raison du caractère parfois ténu des vestiges, du faible niveau de structuration évidente, lorsqu'il existe, et des matériaux d'études considérés, lithiques et plus rarement osseux. Par ailleurs le diagnostic mécanisé nécessite des stratégies particulières avec, comme cas spécifique, celui des sites de milieu humide, comme dans la Somme (Ducrocq, 2010). Deux méthodes de sondage peuvent être recensées, en puits et en tranchées. Elles marquent globalement, pour le Mésolithique, l'opposition entre le Nord, d'une part, et le bassin parisien et l'Est de la France, d'autre part. Ces dernières régions possèdent des plaines alluviales à fort potentiel, dominées par des séquences stratigraphiques holocènes qui dépassent rarement les deux mètres de limons de débordement, hors ou en limite de la nappe aquifère (Ruffey-sur-Seille, Choisey, Pont-sur-Yonne). Ces contextes se prêtent donc assez bien à un diagnostic classique en tranchées.

La phase d'acquisition des données n'oppose pas, dans ses principes méthodologiques, le diagnostic et la fouille; seuls les objectifs les séparent. Un des enjeux est bien d'assurer la pérennité de l'information et l'intégration des données de diagnostic à celles de la fouille. À la nécessité d'assurer le transfert de l'information doit s'ajouter celle de son exploitation optimale, car le passage à la fouille est loin d'être toujours assuré. Il est ainsi possible de renseigner différents aspects des occupations (industriel, économique, palethnographique et autre) et d'alimenter des problématiques archéologiques. En ce sens, le diagnostic de sites mésolithiques constitue un acte scientifique déterminant qui a largement contribué à faire évoluer nos connaissances.

\section{Les occupations de plein air : des réalités très} diverses. Les opérations d'ampleur limitée, réalisées généralement en dehors du cadre préventif, ont eu tendance à donner l'image de campements spatialement très restreints. Si cela correspond à une certaine réalité, la mise au jour de campements très étendus en constitue une autre. Les occupations de Ruffey-sur-Seille, reconnues sur une surface de $25000 \mathrm{~m}^{2}$, de la rue Farman à Paris, sur $10000 \mathrm{~m}^{2}$, ainsi que Dammartin-Marpain, dans le Jura, montrent que nous avons affaire à de vastes portions de plaine occupées. Dès lors, plusieurs questions se posent avec, en premier lieu, celle du rythme de fréquentation (soit la fréquence des séjours) et donc du nombre de campements. Cette question, n'autorise bien souvent que des réponses partielles. Grâce à l'étude assez poussée des occupations de
Ruffey-sur-Seille, nous avons pu préciser la réalité spatiale du campement. Elle s'exprime de manière diverse avec une forme élémentaire constituée d'une seule unité d'activité (Séara, 2008), et une forme plus complexe qui en associe plusieurs. La répétition du premier cas de figure, à l'instar du Mésolithique moyen de Ruffey-sur-Seille, peut donner l'image d'une occupation très extensive, mais qui résulte en fait du cumul de différentes occupations sur près d'un hectare. Bien que la chronologie interne de cette occupation n'ait pas pu être précisée, elle souligne une importante segmentation dans l'espace, qui traduit ici la succession de campements et donc la discontinuité chronologique de l'occupation [Fig.2]. Ce point est caractérisé par un éclatement des secteurs d'occupation assez important, probablement à la faveur d'un milieu favorable à ce scénario.

La seconde forme de campement, est illustrée par l'occupation du Mésolithique ancien de Ruffeysur-Seille (niveau R4) (Séara, 2006) ou encore par celle du site de la rue Farman à Paris [Fig.3]. Le caractère spatialement très délimité des secteurs d'occupation, ainsi que leur configuration, évoquent sans conteste l'existence de plusieurs unités dont l'implantation et le fonctionnement s'avèrent très proches. Les grandes catégories de vestiges sont présentes dans des proportions constantes et se distribuent spatialement de la même manière, ce qui pouvait donner l'impression d'unités autonomes et diachroniques. Ce n'est que grâce à la réalisation des remontages que la contemporanéité d'au moins trois unités a pu être établie, montrant ainsi la possibilité de campements constitués par la répétition d'un même module d'implantation. Le rapprochement spatial ne s'explique pas par une relation de complémentarité fonctionnelle, tout du moins évidente, comme semble l'indiquer la nature des remontages inter-unités, constitués essentiellement d'éclats à Ruffey-sur-Seille et de fragments de plaquette en grès à Farman. Ainsi, la forme de ce campement pourrait traduire une division sociale impliquant des regroupements familiaux ou de toute autre nature.

Le campement : un lieu d'activités multiples. Les différents campements ont constitué le cadre du déroulement de nombreuses activités qui, pour l'essentiel, peuvent nous échapper en l'absence d'investigations tracéologiques. En outre, certaines catégories de restes organiques ou minéraux renvoient directement aux activités qui en sont à l'origine. Nous pensons bien évidemment aux restes de faune qui, lorsqu'ils sont conservés, documentent certains aspects de la chasse, mais également à toute la chaîne de transformation qui en résulte, à savoir le traitement des carcasses et leur consommation. C'est ainsi que certaines parties anatomiques manquantes indiquent très clairement l'import de certains quartiers à des fins et vers des lieux qui nous échappent pour l'essentiel. Les variations de fréquence des espèces identifiées, dont le cerf, le sanglier, le chevreuil et l'aurochs peuvent s'expliquer par les conditions du milieu 


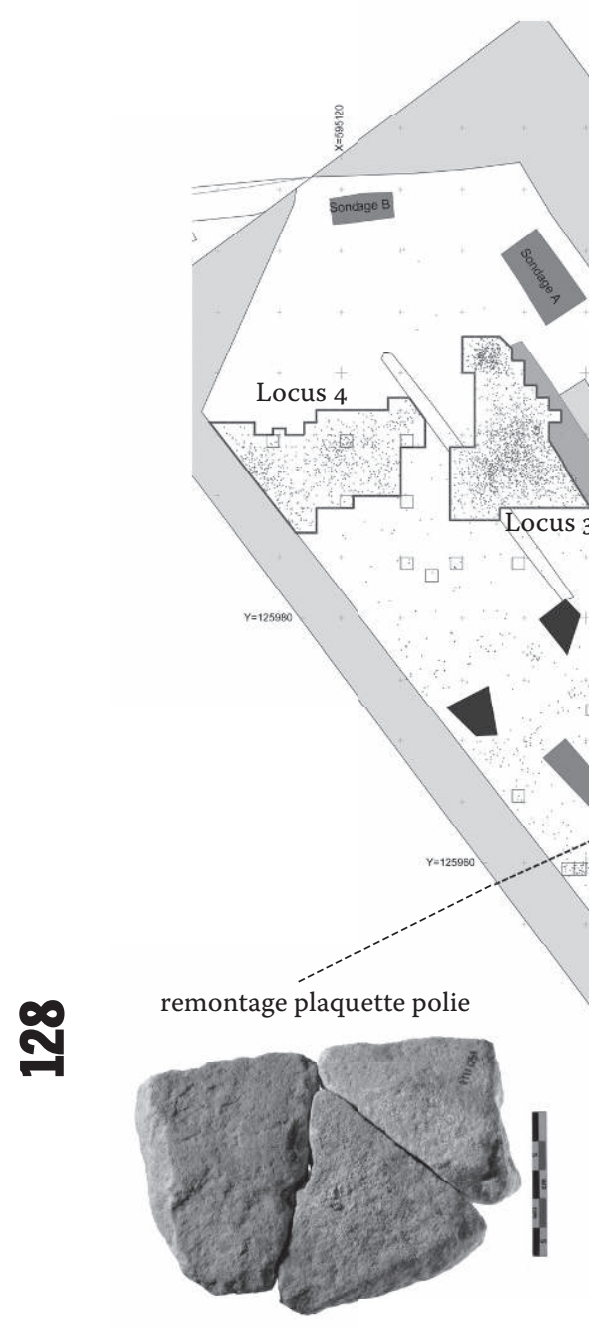

Paris $15^{\text {ème }}-62$ rue Farman"

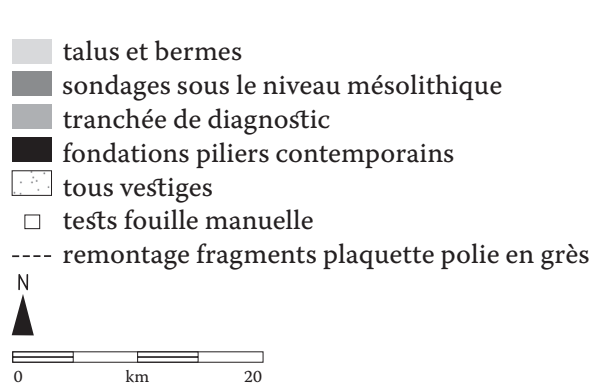

[Fig.3] Paris, Rue Farman. Ce plan de l'ensemble des vestiges mésolithiques montre l'existence de concentrations spatialement bien délimitées. La relation chronologique a pu être précisée grâce à la pratique des remontages, dont celui de fragments d'une même plaquette en grès.
[Fig.4] Ruffey-sur-Seille (Jura). Au sein d'un foyer non aménagé, identifié par de légères zones de sédiment rubéfié et par des vestiges chauffés et brûlés, a été mis au jour un ensemble de 4 armatures, dont 3 triangles isocèles effilés et une pointe à base naturelle en position légèrement décalée. Cet ensemble suggère l'emplacement d'une flèche (brûlée dans le foyer) dont les armatures auraient gardé leur position fonctionnelle. Nous avons ici un exemple exceptionnel qui, tout en traduisant les excellents conditions de conservation, documente la question de l'équipement de chasse appartenant, dans le cas présent, à un groupe sauveterrien.
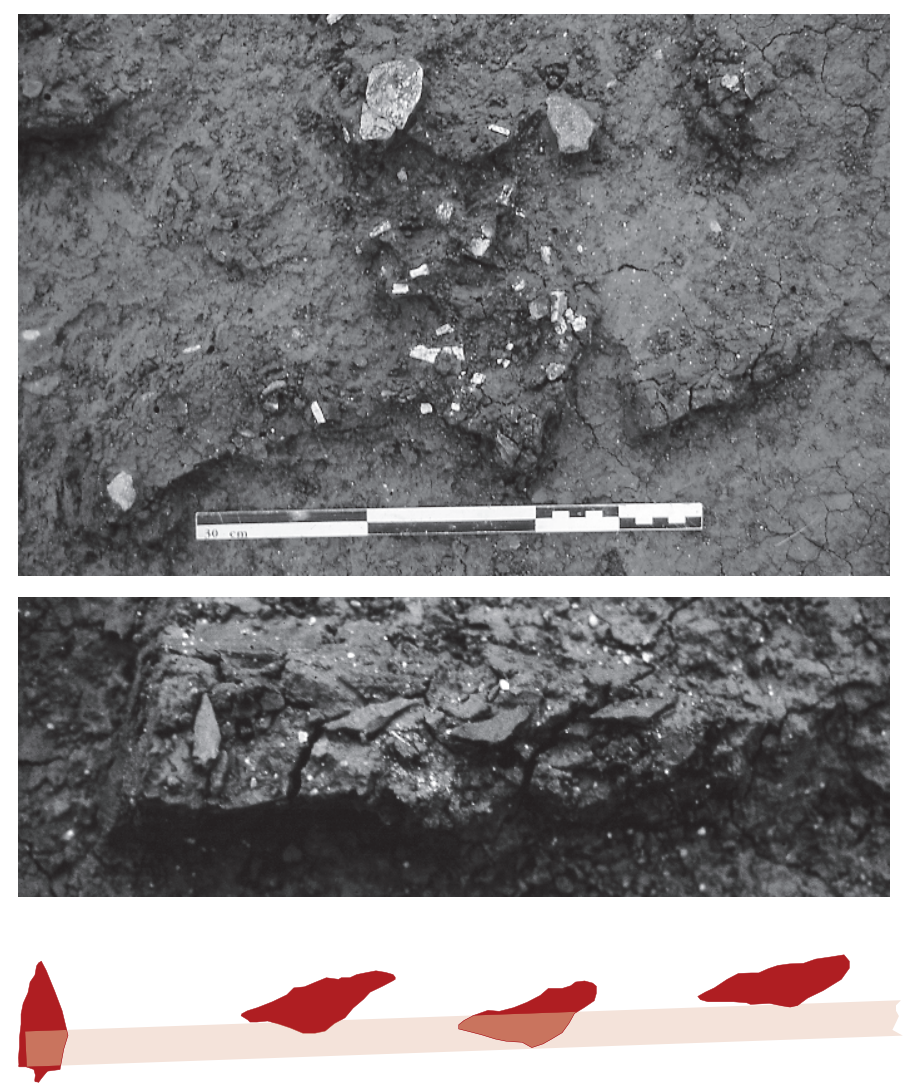
ou refléter la pratique de chasses sélectives ou tout simplement des opportunités d'abattage. C'est ainsi qu'une forme de chasse «spécialisée » ressort de l'étude de l'occupation du Mésolithique récent de Ruffey-sur-Seille, qui a livré de nombreux restes d'aurochs. Il en va de même des nombreux sites beuroniens du Nord de la France qui affichent une certaine prédilection pour le sanglier (Ducrocq, à paraître).

Les activités se rapportant à la chasse impliquent le réarmement des flèches, attesté par les armatures de flèches retrouvées à différents stades, en cours d'élaboration avec des fragments et des déchets caractéristiques, utilisées en portant des traces d'impact diagnostics ou, exceptionnellement, en position fonctionnelle, à l'image des armatures retrouvées dans un foyer à Ruffey-sur-Seille [Fig.4] C'est ainsi que les séries d'armatures collectées peuvent être assez conséquentes comme à Paris, rue Farman, avec 300 individus (Souff, Marti, 2011) et 680 à Dammartin-Marpain dans le Jura (Séara, Roncin, 2010). D'autres éléments en os, beaucoup plus rares, évoquent la chasse, voire la pêche. Il s'agit de pointes comme celles retrouvées à Farman ou à Rueil-malmaison (Lang, 1997 ; Lang, Siccard, 2008).

Toutes les activités de découpe échappent, quant à elles, à l'œil nu, et la tracéologie révèle pour cela la mise en œuvre assez importante d'éclats ou de lamelles bruts qui interviennent aux côtés de grattoirs pour le travail des peaux à différents états, frais et sec. Dans tout cela, rien de très original pour ces populations de chasseurs-cueilleurs, l'originalité étant davantage la mise en évidence de plus en plus fréquente, grâce à la tracéologie, et dans des proportions très variables, du travail de la matière végétale (Gueret, à paraître). Cette source d'analyse indique aussi parfois, comme à Dammartin-Marpain, un travail de matières minérales assez tendres, peut-être à l'image du grès, utilisé en particulier pour la confection de petits polissoirs à rainure.

N'oublions pas l'activité fondamentale sans laquelle très peu d'actions auraient été possibles. Il s'agit de la taille des matières siliceuses à l'origine des différents supports d'outils qu'ils soient retouchés ou non. Cette activité est le plus souvent caractérisée par l'absence de poste de taille clairement identifiable, mais parfois par des amas de débitage modestes, affichant, dans certains cas, un étalement de leurs pièces lithiques, constat que l'on peut expliquer par des actions de tris et de prélèvements en particulier des lamelles [Fig.5]. La pratique des remontages donne des résultats très significatifs qui nous renseignent à la fois sur les aspects techniques et les objectifs recherchés, mais également sur les relations spatiales entre les différents éléments remontés.

Jusqu'à peu encore, la définition des activités ne prenait en compte que la nature des vestiges et les usages implicitement liés à la typologie lithique, ce qui conduisait à conclure presque toujours à une gamme d'activités assez peu diversifiées et tournées presque exclusivement vers la chasse. Mais c'est en fait une assez grande diversité des tâches qui a dominé la vie de ces campements. Les fragments d'ocre, de coquilles de noisettes brûlées, de galets, d'outils massifs en grès ou en silex le corroborent. En revanche certaines activités, comme la pêche, semblent être étonnement absentes au regard de la position de fonds de vallée de la plupart de nos gisements, même si l'on peut imputer en partie ce constat aux difficultés de conservation des équipements.

Malgré toutes ces données, la question du statut de ces occupations se pose encore et rien ne permet d'envisager une véritable hiérarchisation des sites. Les différences constatées seraient bien plus la conséquence de la variabilité de la durée du séjour, qui apparaît généralement courte, allant de quelques jours à quelques semaines tout au plus.

Culture et peuplement. Dans ce domaine, c'est notre capacité à bien cartographier les données du peuplement et leur appartenance culturelle qui se pose en raison d'une réalité très difficile à cerner. Cette difficulté est liée à l'hétérogénéité des données et à leur variabilité selon les régions. L'un des problèmes a consisté à considérer au même niveau des rangs hiérarchiques très différents, impliquant, au sommet de l'arborescence, un territoire social puis des territoires annuels (Clark, 1975). Les travaux conduits par R. Newell vont dans le même sens, en proposant des familles de langage, des tribus ou encore des bandes (Newell et al., 1990). Le niveau le plus bas rappelle les cultures envisagées par J.-G. Rozoy, assimilées à des tribus dialectales (Rozoy, 1998). Si tout cela demeure bien évidemment théorique, l'un des marqueurs privilégiés pour la définition des groupes culturels reste la typologie lithique et, plus spécifiquement, celle des armatures de flèches. D'autres données pourraient jouer un rôle comme l'industrie osseuse, mais les données sont, pour nos régions, encore très limitées.

L'intérêt pour les armatures a très vite porté sur leur charge culturelle, considérée comme très grande, et, pour cette raison, parfois surestimée. En effet, on a tellement caractérisé d'affirmation culturelle leur forme ou leur mode de fabrication, que des divergences minimes, voire insignifiantes, ont été considérées comme des critères suffisants à la distinction de groupes parfois très localisés. Il est probable que ces différences sont davantage liées à la variabilité résultant des paramètres multiples qui entrent dans la fabrication des armatures. Il existe bien une réalité culturelle mais, pour qu'elle soit déclinée à un niveau équivalent au groupe local, il est indispensable de disposer d'une masse de données importantes et homogènes sur de vastes territoires, à l'image de la Somme, par exemple, qui permet d'isoler, au sein du technocomplexe à pointe à base transversale ou Beuronien, un Beuronien à segments (Ducrocq, à paraître). Ce même techno-complexe se retrouve sur une aire géographique très large, englobant une vaste partie de la France, au nord de la Loire. Cette large distribution implique, comme cela se trouve souligné grâce au Beuronien à segments, des différences dans cette vaste entité, ce 

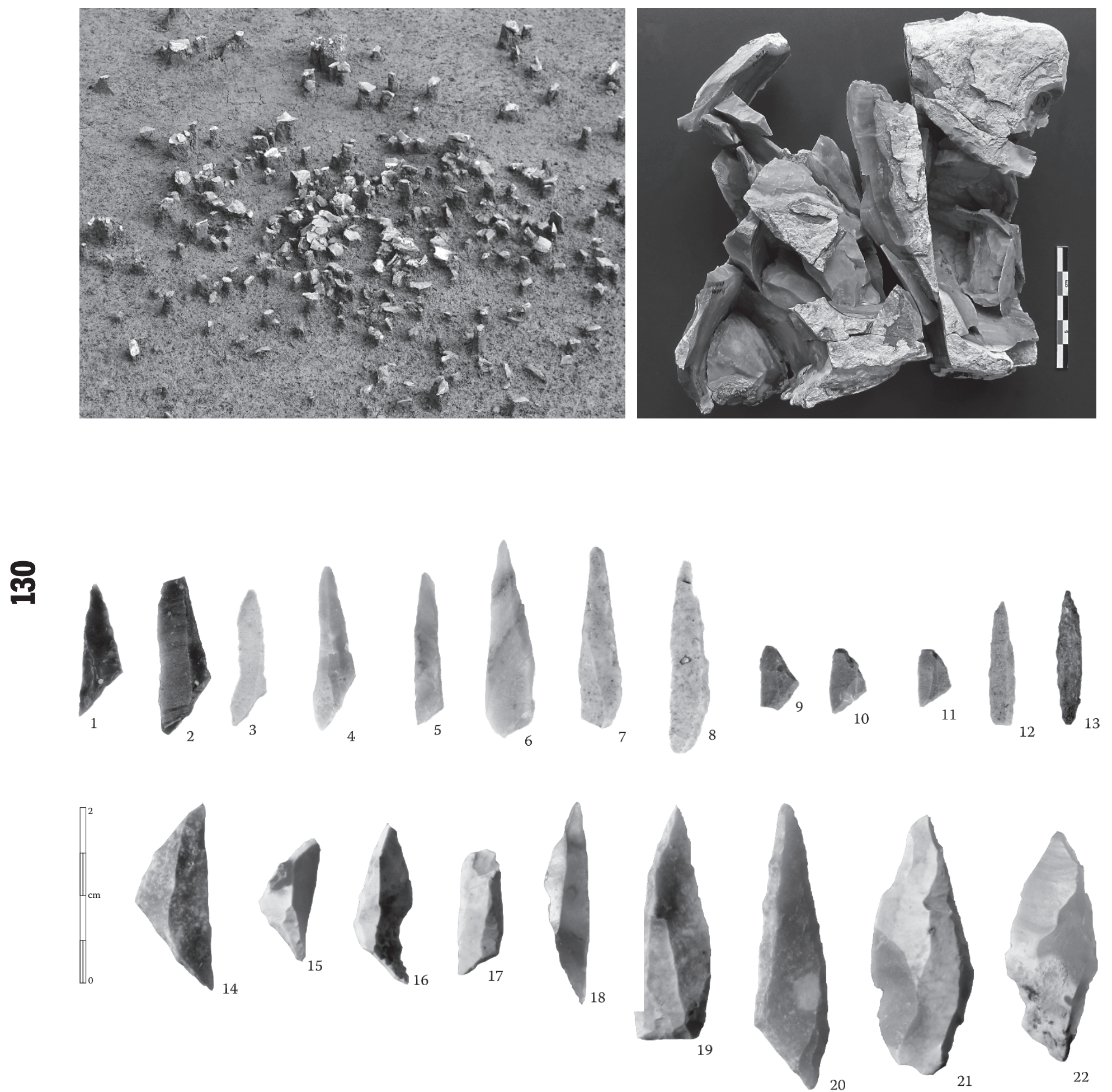

[Fig.6] Dammartin-Marpain (Jura). En haut se trouve un amas de débitage au caractère étalé, ce qui traduit des tris et des prélèvements d'éléments

lithiques. La pratique du remontage, comme cet ensemble remarquable qui associe 91 éléments pour un poids de 74.0 grammes, montre l'existence d'importants vides qui correspondent aux lamelles manquantes. Leur absence du poste de taille indique clairement le prélèvement quasi intégral de ces éléments.
[Fig 7] Armatures de flèches caractéristiques du Sauveterrien (1 à 13) et du Beuronien (14 à 22). Les premières sont principalement constituées de triangles scalènes effilés (1 à 5), de micro-isocèles (9 à 11) et de pointes de Sauveterre (12-13). Les secondes associent, pointe à base transversale (19), triangles isocèles (14-15), segments (18) et triangles scalènes (17 et 20). 
qu'évoquent aussi les données de l'Est de la France avec un Beuronien plus classique, associant des triangles isocèles puis des triangles scalènes [Fig.6]. Le pendant culturel méridional est constitué par le Sauveterrien, qui se caractérise par un cortège d'armatures composé de triangles isocèles puis scalènes au caractère effilé associés à des pointes fusiformes appelées «pointes de Sauveterre ». Cette dualité culturelle domine tout au long du premier Mésolithique, entre 8500 et 6500 avant notre ère, avec, en apparence, le maintien d'une plus forte cohésion pour la partie méridionale. En effet, aux alentours de 7500 avant notre ère, la partie nord se distingue par une mosaïque de groupes soit d'obédience beuronienne, soit en rupture, à l'image du groupe à feuille de gui ou $\mathrm{du}$ sauveterrien à denticules du bassin parisien.

Entre ces deux zones, se trouve une bande médiane dont les industries ont pour particularité d'associer des caractères sauveterriens et beuroniens, ce qui a conduit à rattacher ces industries à une entité spécifique le Ligérien. La difficulté est d'arriver à évaluer si la mixité culturelle donnée par les armatures s'accorde avec une seule et même industrie, ou bien résulte d'une réoccupation des mêmes sites par des groupes culturellement distincts comme cela a été démontré à Ruffey-sur-Seille. Ce cas de figure est fréquent et la fouille récente du site de Dammartin-Marpain a montré la juxtaposition, sur un même lieu, d'occupations de chronologie et d'appartenance culturelle différentes. L'un des handicaps majeurs du chercheur résulte souvent du contexte de conservation de la plupart des gisements de cette zone géographique, avec des occupations conservées dans des milieux sableux : Ingrandes-de-Touraine (Lang, Kildéa, 2007), SaintRomain-sur-Cher (Kildéa, 2008a) Mareuil-surCher (Kildéa, 2008b) etc. Cela ne permet pas de trancher entre homogénéité et hétérogénéité des séries. La réflexion portant sur la définition des groupes culturels, leur emprise géographique et leurs possibles interactions doit s'appuyer avant tout sur des sites aux données bien assurées, à l'image du Nord ou de l'Est de la France. Certes, ces données ne sont pas toujours transposables, mais elles soulignent la nécessité de prendre en compte la complexité et la variété des schémas d'occupations mésolithiques, au risque de conclure à des attributions culturelles erronées ou sans réels fondements.

Perspectives. Le renouveau des données permet d'envisager de multiples perspectives d'étude qui, pour la plupart, s'inscrivent dans le long terme, ce qui n'est pas sans poser des problèmes dans un contexte d'archéologie préventive, par définition contrainte tant en durée qu'en moyens. En effet, la nécessité de prolonger des études parfois à peine esquissées dans le cadre des rapports d'opérations, se heurte bien souvent à cette réalité. Malgré le caractère encore modeste de la communauté des mésolithiciens, force est de constater l'affirmation d'un dynamisme soutenu qui s'exprime par des publications régulières et par des échanges constants avec la communauté scientifique dans le cadre de manifestations scientifiques, au sein des UMR et du milieu universitaire. La part plus importante des opérations préventives durant ces dernières années explique partiellement ce renouveau, majoritairement impulsé par le dynamisme des équipes impliquées, ce qui conduit à considérer la recherche sur le Mésolithique comme l'une de celles dont l'évolution a été la plus significative.

Sur un plan méthodologique opérationnel, les protocoles de détection et de caractérisation sont désormais bien validés, mais il serait nécessaire d'en améliorer les principes de mise en œuvre, en particulier, face aux contraintes très fortes imposées par les occupations situées en deçà de la nappe aquifère, à l'image de la Picardie, mais probablement d'un nombre assez important de vallées alluviales. Cet objectif renvoie à des gisements extrêmement bien conservés, à la faveur de milieu humide, à l'image du site de Noyen-surSeine (Mordant, 1989)

La caractérisation spatiale des campements bénéficie de données de plus en plus nombreuses mais qui, fait assez récent pour l'étude du Mésolithique, sont discutées sur le plan de leur fiabilité à partir des contextes de gisement. Ce préalable est indispensable à toute étude et, bien évidemment, à celle de l'organisation spatiale. En effet, parmi les principaux objectifs à atteindre, celui de la justification des différentes formes de structuration spatiale doit s'appuyer sur un contexte archéologique au caractère sûr et démontré. Cette étape sera facilitée par la multiplication des données et, actuellement, elle ouvre davantage sur un questionnement large que sur de véritables réponses. Mais des pistes se dessinent. Deux niveaux peuvent être distingués dans cette réflexion future : celui, strict, du campement et celui, plus large, du système des différentes occupations. C'est ainsi que l'étude fonctionnelle de l'industrie lithique aura un rôle déterminant à jouer car, tout en jetant un éclairage sur les activités et la vocation des campements, elle contribue à préciser la nature des relations entre les gisements (complémentarité ou répétition); la forme de la structuration spatiale pourrait y répondre. D'autres domaines de connaissances touchant les économies de chasse ou encore de transformation des matières premières siliceuses ont été considérablement améliorés. En effet, les schémas techniques du débitage et leurs objectifs ont été efficacement renseignés par une pratique toute récente pour l'étude du Mésolithique, celle des remontages. Le domaine du funéraire n'est pas en reste, mais les données sont encore faibles pour tirer des généralités, d'autant plus qu'il existe une très grande variabilité dans les pratiques, tant de l'inhumation que de la crémation.

Les nombreux acquis à caractère palethnographique, pour l'essentiel issus des fouilles préventives, permettent, conjointement à la masse considérable de données tirées des opérations programmées, de rendre proche l'échéance d'une véritable analyse palethnologique. 
Pour cela, il est encore nécessaire d'étoffer le corpus de sites et de tenter de rééquilibrer les disparités régionales en mettant à profit une dynamique désormais bien affirmée.

BOSTYN F., SÉARA F., 2011 : Occupations de plein air mésolithique et néolithique : Le site de la Presle à Lhéry dans la Marne, S.P.F, Travaux 10, $287 \mathrm{p}$.

CLARK J.G.D., 1975 : The earlier stone age settlement of Scandinavia, Cambridge, Cambridge University Press, $282 \mathrm{p}$.

Ducroce T. 2001 : Le Mésolithique du Bassin de la Somme. Insertion dans un cadre morphostratigraphique, environnemental et culturel, publications du C.E.R.P., $n^{\circ} 7$, Lille, 253 p., 200 fig.

Ducrocq T., Bridault A., Coutard S., 2008 : « Le gisement mésolithique de Warluis (Oise) : approche préliminaire », in FAGNART J.-P. et al., p. 85-106.

DUCROCQ T., 2010 : "Quelques exemples dans le bassin hydrographique de la Somme ", in Le diagnostic des sites paléolithiques et mésolithiques, Actes du séminaire des 5-6 décembre 2006, Cahier de l'Inrap, $n^{\circ} 3$, p. $35-48$.

DUCROCQ T., à paraître : « Le Beuronien à segments dans le Nord de la France. Prémices d'une approche palethnologique », in VALENTIN B. et al.

Fagnart J.-P., Thévenin A., Ducrocq T., Souffi B., Coudret P. (Dir), 2008 : Le début du Mésolithique en Europe du Nord-Ouest, Actes de la table ronde d'Amiens, 9-10 octobre 2004, Paris, éd. Société préhistorique française (Mémoire XLV).

GUERET C., à paraître : «Identité et variabilité de l'outillage lithique du Premier Mésolithique en Belgique et dans le Nord de la France : Les apports de l'analyse fonctionnelle », in VALENTIN B.et al.

Hinout J., 1990 : «Évolution des cultures épipaléolithiques et mésolithiques dans le Bassin Parisien », Revue Archéologique de Picardie, ${ }^{\circ} 3 / 4$, p. 5-14.

KILDEA F., 2008a : « Les occupations du Mésolithique ancien et moyen de Saint-Romain-sur-Cher (Loir-et-Cher) », in FAgnarT J. P. et al., p. $153-167$.

KILDÉA F. (DIR.), 2008b : «La Croix de Bagneux » à Mareuil-sur-Cher (Loir-et-Cher) (A85, section $M_{3}$, site 30$)$. Un site paléolithique à occupations multiples dans la vallée du Cher, Rapport final d'opération de fouille, Inrap, $643 \mathrm{p}$.

LANG L., 1997: Occupations mésolithiques dans la moyenne vallée de la Seine. Rueil-Malmaison «Les Closeaux » (Hauts de Seine), Document Final de Synthèse, Afan A86, SRA Ile-de-France, 2 vol., 384 p.

LANG L., KILDÉA F., 2007: A85, K3, Ingrandes-de-Touraine, site F, « La prairie d'Ingrandes » (Indre-et-Loire), Rapport final l'opération de fouille, Inrap, $88 \mathrm{p}$.

LANG L., SICARD S., 2008 : « Les occupations mésolithiques des Closeaux à Rueil-Malmaison (Hauts-de-Seine) ", in FAGNART J. P. et al., p. 65-83.

Mordant C., Mordant D., 1989 : « Noyen-sur-Seine, site mésolithique en milieu humide fluviatile ", in L'homme et l'eau au temps de la Préhistoire, Actes du 112e congrès national des sociétés savantes, Lyon, 1987, p. 33-52.

Newell R., Kielman D., Constandse-Westermann T.S., Van Der SANDEN W.A.B., VAN GIJN A., 1990 : An inquiry into the ethnic resolution of mesolithic regional groups. The study of their decorative ornaments in time and space, Leiden, Brill $488 \mathrm{p}$.

Rozoy J.-G., 1978 : Les derniers chasseurs. L'Épipaléolithique en France et en Belgique, Mémoire de la Société d'Archéologie Champenoise, 3 vol., 1256 p., 294 fig, 259 pl., 81 tabl.

Rozoy J.-G., 1998 : « Stratégies de chasse et territoires tribaux au Mésolithique ", Bulletin de la Société Préhistorique Française, 95, 4, p. 525-536

SÉARA F., Rotillon S., Cupillard C. (DIR.), 2002 : Campement mésolithiques en Bresse jurassienne; Choisey et Ruffey-sur-Seille (Jura), Paris, MSH (DAF, 92), 344 p.

SÉARA F., 2006 : « Elements of reflection about habitat structural parts from spatial analysis of open air encampments in eastern France : The examples of Ruffey-sur-seille and Choisey (Jura) and Pont-surYonne (Yonne) », in Claus-JoACHIM K. (ÉD.), After the Ice Age, Rottenburg, p. 277-283, 3 fig

SÉARA F., 2008: Campements mésolithique de plein-air : Détection, Caractérisation, Modélisation. De Ruffey-sur-Seille et Choisey (Jura), aux occupations des IX $X^{e}$ et $V I I I^{e}$ millénaires de Pont-sur-Yonne (Yonne), Thèse de doctorat, Université de Bourgogne, 316 p. 26o fig.

SÉARAF., Roncin O., 2010 : Dammartin-Marpain (Jura), Prairie du Milieu. Nouvelles données sur le peuplement mésolithique, néolithique, protohistorique et antique de la basse vallée de l'Ognon, Rapport Final d'Opération, Inrap, 2 vol., 724 p.

Souffi B., MARTi F., 2011 : Paris $15^{e}$ arrondissement, 62 rue Henry Farman. Évolution culturelle et environnementale d'un site stratifié en bord de seine, du Mésolithithique au premier âge du Fer, Rapport final d'opération, Inrap, 4 vol., 1337 p.

Thévenin A., 2008: «Le Mésolithique ancien et moyen de la moitié nord de la France : les grandes lignes du peuplement », in FAGNART J.P. et al., p. 31-50.

Valentin B., Souffi B., Ducroce T., Fagnart J.-P., Séara F., Verjeux C. (DIR), à paraître: Palethnographie du Mésolithique: Recherches sur les habitats de plein air dans la moitié septentrionale de la France et ses marges, Actes de la table-ronde de Paris, 26-27 novembre 2010, Paris, Société préhistorique française
Une connaissance de l'âge du Bronze transfigurée par l'archéologie préventive

Patrice Brun

Université Paris I Panthéon-Sorbonne, UMR 7041 «Archéologie des Sciences de l'Antiquité "

Cyril Marcigny

Inrap, UMR 6566 CReAAH

$\mathbf{N}$ otre connaissance de l'âge du Bronze est longtemps restée bridée par l'insuffisance des moyens de recherche sur le terrain.

Il a d'abord fallu attendre la mise en œuvre de prospections et de fouilles de grandes surfaces pour que le tableau historique et sociologique de cette période charnière se précise. Mais une autre condition a été tout aussi indispensable : la multiplication des opérations rendant possible la mise en évidence des réseaux locaux, voire régionaux, de sites en interaction. Ce double changement d'échelle de la recherche archéologique a déjà produit des résultats spectaculaires : il augure des progrès décisifs sur l'histoire de notre pays dans la longue durée.

\section{Lâge du Bronze, à la charnière de 4300 ans longtemps mal documentés. La connaissance} de la tranche de temps qui s'intercale entre les périodes paléolithique et romaine en France est longtemps restée fondée sur des découvertes de tombes et de dépôts non funéraires. À propos des sites d'habitat, on savait bien que certains avaient été fortifiés lorsque leur rempart, encore visible, avait fait l'objet de sondages permettant de le dater, mais l'architecture des bâtiments et l'organisation d'ensemble de ces établissements nous échappaient presque totalement. Quelques établissements, localisés en bord de lac et, par conséquent, mieux conservés, avaient certes fourni de précieuses indications, grâce à des fouilles d'envergure exceptionnelle, en Allemagne au Wasserburg Buchau (Reinerth, 1928), ou en Grande-Bretagne à Little Woodbury (Bersu, 1940), par exemple, mais, presque partout ailleurs, l'absence de bois conservés et l'exigüité des sondages archéologiques entretenait les préjugés primitivistes à l'égard des sociétés en question. Ainsi, malgré ces beaux exemples d'agglomérations durables, aux solides et vastes constructions en bois, terre et chaume ou joncs, on a longtemps persisté à expliquer la faiblesse des traces d'habitat par la légèreté du bâti : on envisageait des huttes construites pour de courtes occupations en raison d'un mode de vie fondamentalement nomade.

Dubitatifs sur ce point, quelques archéologues ont pu profiter du cadre légal et financier plus favorable mis en place dans leur pays pour initier des fouilles utilisant des décapages en aire ouverte sur de grandes surfaces. Des résultats spectaculaires n'ont alors pas tardé à transformer la connaissance des sites d'habitat de cette période-clé initiée par la sédentarisation graduelle qu'a rendue possible l'adoption d'une économie agro-pastorale et qui s'est achevée avec l'émergence de l'État avec ses principaux corolaires, la ville et l'écriture. L'agencement des trous des poteaux porteurs a 